BULLETIN (New Series) OF THE

AMERICAN MATHEMATICAL SOCIETY

Volume 40, Number 4, Pages 429-440

S 0273-0979(03)00992-3

Article electronically published on July 17, 2003

\title{
ON A THEOREM OF JORDAN
}

\author{
JEAN-PIERRE SERRE
}

\begin{abstract}
The theorem of Jordan which I want to discuss here dates from 1872. It is an elementary result on finite groups of permutations. I shall first present its translations in Number Theory and Topology.
\end{abstract}

\section{Statements}

1.1. Number theory. Let $f=\sum_{m=0}^{n} a_{m} x^{m}$ be a polynomial of degree $n$, with coefficients in $\mathbf{Z}$. If $p$ is prime, let $N_{p}(f)$ be the number of zeros of $f$ in $\mathbf{F}_{p}=\mathbf{Z} / p \mathbf{Z}$.

Theorem 1. Assume

(i) $n \geq 2$,

(ii) $f$ is irreducible in $\mathbf{Q}[x]$. Then

(a) There are infinitely many $p$ 's with $N_{p}(f)=0$.

(b) The set $P_{0}(f)$ of $p$ 's with $N_{p}(f)=0$ has a density $c_{0}=c_{0}(f)$ which is $>0$.

[Recall that a subset $P$ of the set of primes has density $c$ if

$$
\lim _{X \rightarrow \infty} \frac{\text { number of } p \in P \text { with } p \leq X}{\pi(X)}=c,
$$

where $\pi(X)$ is as usual the number of primes $\leq X$.]

Moreover,

Theorem 2. With the notation of Theorem 1, one has $c_{0}(f) \geq \frac{1}{n}$, with strict inequality if $n$ is not a power of a prime.

Example. Let $f=x^{2}+1$. One has $p \in P_{0}(f)$ if and only if $p \equiv-1(\bmod 4)$; this set is well-known to have density $1 / 2$. We shall see more interesting examples in $₫ 5$

1.2. Topology. Let $\mathbf{S}_{1}$ be a circle.

Let $f: T \rightarrow S$ be a finite covering of a topological space $S$. Assume:

(i) $f$ has degree $n$ (i.e. every fiber of $f$ has $n$ elements), with $n \geq 2$,

(ii) $T$ is arcwise connected and not empty.

Theorem 3. There exists a continuous map $\varphi: \mathbf{S}_{1} \rightarrow S$ which cannot be lifted to the covering $T$ (i.e. there does not exist any continuous map $\psi: \mathbf{S}_{1} \rightarrow T$ such that $\varphi=f \circ \psi)$.

Received by the editors March 1, 2003

2000 Mathematics Subject Classification. Primary 06-XX, 11-XX, 11F11.

This text first appeared in Math Medley 29 (2002), 3-18. The writing was done with the help of Heng Huat Chan. (C)2002 Singapore Mathematical Society. Reprinted with permission. 
1.3. Finite groups. Let $G$ be a group acting on a finite set $X$. Put $n=|X| 1$

Theorem 4 (Jordan [9]). Assume that

(i) $n \geq 2$,

(ii) $G$ acts transitively on $X$.

Then there exists $g \in G$ which acts on $X$ without fixed point.

Assume that $G$ is finite (which is the case if $G$ acts faithfully on $X$ ). Let $G_{0}$ be the set of $g \in G$ with no fixed point. Call $c_{0}$ the ratio $\frac{\left|G_{0}\right|}{|G|}$.

Theorem 5 (Cameron-Cohen [4]). One has $c_{0} \geq \frac{1}{n}$. Moreover, if $n$ is not a power of a prime, $c_{0}>\frac{1}{n}$.

\section{Proofs of the Group theoretical Statements}

2.1. Burnside's Lemma. Let $G$ be a finite group acting on a finite set $X$. If $g \in G$, let $\chi(g)$ be the number of fixed points of $g$ on $X$, i.e. $\chi(g)=\left|X^{g}\right|$.

Burnside's Lemma (cf. [6, §4.2], [3, §145]). The number of orbits of $G$ in $X$ is equal to

$$
\langle\chi, 1\rangle=\frac{1}{|G|} \sum_{g \in G} \chi(g)=\int \chi .
$$

(If $\varphi$ is a function on $G$, and $S$ is a subset of $G$, we denote by $\int_{S} \varphi$ the number $\frac{1}{|G|} \sum_{g \in S} \varphi(g)$. When $S=G$, we write $\int \varphi$ instead of $\int_{G} \varphi$.)

By decomposing $X$ into orbits, it is enough to prove the lemma for $X \neq \emptyset$ and $G$ transitive on $X$, i.e. $X \simeq G / H$ for some subgroup $H$ of $G$.

We give three proofs, in different styles.

First Proof: "Analytic Number Theory Style".

$$
\begin{aligned}
\sum_{g \in G} \chi(g) & =\sum_{g \in G} \sum_{\substack{x \in X \\
g \cdot x=x}} 1 \\
& =\sum_{x \in X} \sum_{\substack{g \in G \\
g \cdot x=x}} 1 \\
& =\sum_{x \in X}|H|=|H| \cdot|X|=|G| .
\end{aligned}
$$

Second Proof: "Combinatorics Style". Let $\Omega \subset G \times X$ be the set of pairs $(g, x)$ with $g \cdot x=x$. We compute $|\Omega|$ by projecting on each factor. In the projection $\Omega \rightarrow G$, the fiber of $g \in G$ has $\chi(g)$ elements and hence

$$
|\Omega|=\sum_{g \in G} \chi(g) .
$$

On the other hand, in the projection $\Omega \rightarrow X$, the fiber of $x \in X$ is a conjugate of $H$ and hence

$$
|\Omega|=\sum_{x \in X}|H|=|H| \cdot|G / H|=|G| .
$$

\footnotetext{
${ }^{1}$ If $S$ is a finite set, we denote by $|S|$ the number of elements of $S$.
} 
Third Proof: "Algebra Style". The function $\chi$ is the character of the permutation representation defined by $X$. Hence, $\langle\chi, 1\rangle$ is the dimension of the space of $G$-invariant elements of that representation, which is obviously 1 .

\subsection{Proof of Theorem 5 .}

Lemma. $\int \chi^{2} \geq 2$.

First Proof (by Burnside's Lemma). If $g \in G, \chi^{2}(g)$ is the number of points of $X \times X$ fixed by $g$ and $\int \chi^{2}$ is the number of orbits of $G$ on $X \times X$, which is $\geq 2$, as one sees by decomposing $X \times X$ into the diagonal and its complement.

This also shows that $\int \chi^{2}=2$ if and only if $G$ is doubly transitive on $X$.

Second Proof (by Group Representations). We have $\chi=1+\chi^{\prime}$, where $\chi^{\prime}$ is a nonzero real character with $\int \chi^{\prime}=0$. Therefore,

$$
\int \chi^{2}=1+\int \chi^{\prime 2} \geq 2
$$

with equality if and only if $\chi^{\prime}$ is irreducible.

We now prove Theorem [5. Recall that $G_{0}$ is the set of $g \in G$ with $\chi(g)=0$. If $g \notin G_{0}$, we have $1 \leq \chi(g) \leq n$ and therefore

$$
(\chi(g)-1)(\chi(g)-n) \leq 0 .
$$

Hence,

i.e.,

$$
\int_{G-G_{0}}(\chi(g)-1)(\chi(g)-n) \leq 0
$$

$$
\int_{G}(\chi(g)-1)(\chi(g)-n) \leq \int_{G_{0}}(\chi(g)-1)(\chi(g)-n)=n \int_{G_{0}} 1 .
$$

The right hand side is

$$
n \int_{G_{0}} 1=n c_{0}
$$

and the left hand side is

$$
\int_{G}\left(\chi^{2}-(n+1) \chi+n\right) .
$$

By the lemma, and the fact that $\int \chi=1$, we have

$$
\int_{G}\left(\chi^{2}-(n+1) \chi+n\right) \geq 2-(n+1)+n=1,
$$

hence

$$
1 \leq n c_{0} .
$$

2.3. Equality in Theorem 5. The proof of Theorem 5 shows that equality holds if and only if $\int \chi^{2}=2$ and $(\chi(g)-1)(\chi(g)-n)=0$ for every $g \in G-G_{0}$, i.e. if and only if $G$ is doubly transitive and no element of $G-\{1\}$ fixes 2 points. By a theorem of Frobenius [8], the set $N=\{1\} \cup G_{0}$ is then a normal subgroup of $G$, and $G$ is a semidirect product: $G=H \cdot N$. Hence, $|N|=n$, and $(n-1) /|G|=\left|G_{0}\right| /|G|=c_{0}=1 / n$, i.e. $|G|=n(n-1),|H|=n-1$. Moreover, the action of $H$ on $N-\{1\}$ by conjugation is a free action. Since $H$ and $N-\{1\}$ have the same number of elements, one sees that $H$ acts freely and transitively on $N-\{1\}$. This implies that $N$ is a $p$-group for some prime $p$ (and even more: $N$ is an elementary abelian $p$-group). Hence, $n$ is a power of a prime. 
Remarks.

1. It is only for convenience that we have used Frobenius's Theorem [8]. It is possible to give a direct proof, as was already done in Jordan's paper [9].

2. Conversely, if $n$ is a power of a prime, there exists a pair $(G, X)$ with $|X|=n$ and $c_{0}=1 / n$ : take $X=k$, a finite field with $n$ elements, and define $G$ as the group of affine transformations $x \mapsto a x+b$ with $a \in k^{*}, b \in k$.

\section{Proof of the Covering SPACE Statement}

With the same notation as in $\S 1.2$, choose a point $s \in S$. Let $X=f^{-1}(s)$ be the fiber of $s$. Let $G=\pi_{1}(S, s)$ be the fundamental group of $S$ at the point $s$. There is a natural action of $G$ on $X$, and the hypothesis that $T$ is arcwise connected implies that every two points in $X$ can be connected by a path and hence $G$ acts transitively on $X$. Since $n=|X| \geq 2$, Theorem 4 shows that there exists $g \in G$ which has no fixed point on $X$. If we represent $g$ by a loop

$$
\varphi:\left(\mathbf{S}_{1}, s_{0}\right) \rightarrow(S, s),
$$

where $s_{0}$ is a chosen point in $\mathbf{S}_{1}$, then $\varphi$ cannot be lifted to $T$. Indeed, if $\psi: \mathbf{S}_{1} \rightarrow T$ were a lift of $\varphi$, the point $x=\psi\left(s_{0}\right)$ would be a fixed point of $g$.

\section{Proof of the number theoretic statement}

We now prove Theorems 1 and 2 with the help of Theorems 4 and 5 Let $x_{1}, \ldots, x_{n}$ be the roots of $f$ in an algebraic closure $\overline{\mathbf{Q}}$ of $\mathbf{Q}$. Let $E=\mathbf{Q}\left(x_{1}, \ldots, x_{n}\right)$ and let $G=$ Aut $E=$ the Galois group of $E / \mathbf{Q}$. The action of $G$ on the set $X=\left\{x_{1}, \ldots, x_{n}\right\}$ is transitive since $f$ is irreducible over $\mathbf{Q}$. Let $G_{0}$ be the subset of $G$ having no fixed points. By Theorems 4 and 5 , we have

$$
\frac{\left|G_{0}\right|}{|G|} \geq \frac{1}{n}
$$

Let us define a finite set $S$ of "bad" prime numbers, namely, those which divide the discriminant of $f$ or divide the coefficient of $x^{n}$. Assume now that $p \notin S$. Then the reduction $f_{p}$ of $f$ modulo $p$ is a polynomial of degree $n$, whose $n$ roots (in an algebraic closure $\overline{\mathbf{F}}_{p}$ of $\mathbf{F}_{p}$ ) are distinct. Let $X_{p}$ be the set of such roots. We may identify $X_{p}$ and $X$ in the following way:

Let $R=\mathbf{Z}\left[x_{1}, x_{2}, \ldots, x_{n}\right]$ be the ring generated by the $x_{i}$ 's. Choose a homomorphism $\varphi: R \rightarrow \overline{\mathbf{F}}_{p}$ (such a homomorphism exists since $p \nmid a_{0}$, and any other such homomorphism is of the form $\varphi \circ s$, with $s \in G$ ). Such a $\varphi$ defines a bijection $\varphi_{p}: X \rightarrow X_{p}$, which is well-defined up to an element of $G$. Let $\pi_{p}$ be the Frobenius automorphism of $\overline{\mathbf{F}}_{p}$, i.e., $\lambda \mapsto \lambda^{p}$. The map $\pi_{p}$ acts on $X_{p}$. If we identify $X_{p}$ with $X$ via $\varphi_{p}$, we get a permutation $\sigma_{p}$ of $X$ (depending on the choice of $\varphi$ ). One proves that this permutation belongs to $G$. It is called the Frobenius substitution of $p$ (relative to the choice of $\varphi$ ); it is well-defined up to inner conjugation in $G$. We have

$$
\text { If } p \notin S, N_{p} \text { is the number of } x \in X \text { fixed by } \sigma_{p} \text {. }
$$

This follows from the corresponding fact for $X_{p}$ and $\pi_{p}$. (More generally, if $\sigma_{p}$ is a product of disjoint cycles of lengths $l_{\alpha}$, then $f_{p}$ decomposes into a product of $\mathbf{F}_{p}$-irreducible polynomials of degrees $l_{\alpha}$.) Hence, $N_{p}=0$ if and only if $\sigma_{p} \in G_{0}$, where $G_{0}$ is the set of $s \in G$ which acts on $X$ without fixed point. Note that $G_{0}$ is stable under conjugation so that " $\sigma_{p} \in G_{0}$ " makes sense. 
We now recall Chebotarev's Density Theorem (see Notes for Part 4):

Chebotarev's Density Theorem ([19, [1]). Let $C$ be a subset of $G$, stable under conjugation (i.e. a union of conjugacy classes). Then the set $P_{C, S}$ of primes $p \notin S$ with $\sigma_{p} \in C$ has a density, which is equal to $\frac{|C|}{|G|}$.

Applying this theorem to the case $C=G_{0}$ shows that the set $P_{0}(f)$ of Theorem 1 has density $c_{0}=\frac{\left|G_{0}\right|}{|G|}$; by Theorems 4 and 5 this completes the proofs of Theorems 17and 2.

\section{EXAmple: $N_{p}(f)$ FOR $f=x^{n}-x-1$}

5.1. In this section, we consider the special case of $f=x^{n}-x-1, n \geq 2$, and we relate the numbers $N_{p}(f)$ to the coefficients of suitable power series. We limit ourselves to stating the results; for the proofs, see the hints given in the Notes.

Here is a small table of $N_{p}(f)$ for $f=x^{n}-x-1, n=2,3,4,5$ :

\begin{tabular}{|c|c|c|c|c|}
\hline$p$ & $n=2$ & $n=3$ & $n=4$ & $n=5$ \\
\hline 2 & 0 & 0 & 0 & 0 \\
\hline 3 & 0 & 0 & 0 & 0 \\
\hline 5 & 1 & 1 & 0 & 0 \\
\hline 7 & 0 & 1 & 1 & 0 \\
\hline 11 & 2 & 1 & 1 & 0 \\
\hline 13 & 0 & 0 & 1 & 0 \\
\hline 17 & 0 & 1 & 2 & 2 \\
\hline 19 & 2 & 1 & 0 & 1 \\
\hline 23 & 0 & 2 & 1 & 1 \\
\hline$\cdots$ & $\cdots$ & $\cdots$ & $\cdots$ & $\cdots$ \\
\hline 59 & 2 & 3 & 1 & 0 \\
\hline$\cdots$ & $\cdots$ & $\cdots$ & $\cdots$ & $\cdots$ \\
\hline 83 & 0 & 1 & 4 & 0 \\
\hline
\end{tabular}

5.2. The case $n=2$. The discriminant of $f=x^{2}-x-1$ is 5 ; the polynomial $f$ has a double root $\bmod 5$; hence $N_{5}(f)=1$. For $p \neq 5$, we have

$$
N_{p}(f)=\left\{\begin{array}{lll}
2 & \text { if } p \equiv \pm 1 & (\bmod 5) \\
0 & \text { if } p \equiv \pm 2 & (\bmod 5)
\end{array}\right.
$$

If one defines a power series $F(q)=\sum_{m=0}^{\infty} a_{m} q^{m}$ by

$$
F=\frac{q-q^{2}-q^{3}+q^{4}}{1-q^{5}}=q-q^{2}-q^{3}+q^{4}+q^{6}-q^{7}-q^{8}+q^{9}+\cdots,
$$

the above formula can be restated as

$$
N_{p}(f)=a_{p}+1 \quad \text { for all primes } p .
$$

Note that the coefficients of $F$ are strongly multiplicative: one has $a_{m m^{\prime}}=a_{m} a_{m^{\prime}}$ for every $m, m^{\prime} \geq 1$. The corresponding Dirichlet series $\sum_{m=1}^{\infty} a_{m} m^{-s}$ is the $L$-series $\prod_{p}\left(1-\left(\frac{\bar{p}}{5}\right) p^{-s}\right)^{-1}$. 
5.3. The case $n=3$. The discriminant of $f=x^{3}-x-1$ is -23 ; the polynomial $f$ has a double root and a simple root $\bmod 23$; hence $N_{23}(f)=2$. For $p \neq 23$, one has:

$$
N_{p}(f)= \begin{cases}0 \text { or } 3 & \text { if }\left(\frac{p}{23}\right)=1 \\ 1 & \text { if }\left(\frac{p}{23}\right)=-1 .\end{cases}
$$

Moreover, in the ambiguous case where $\left(\frac{p}{23}\right)=1, p$ can be written either as $x^{2}+$ $x y+6 y^{2}$ or as $2 x^{2}+x y+3 y^{2}$ with $x, y \in \mathbf{Z}$; in the first case, one has $N_{p}(f)=3$; in the second case, one has $N_{p}(f)=0$. (The smallest $p$ of the form $x^{2}+x y+6 y^{2}$ is $59=5^{2}+5 \cdot 2+6 \cdot 2^{2}$, hence $N_{59}(f)=3$; cf. table above.)

Let us define a power series $F=\sum_{m=0}^{\infty} a_{m} q^{m}$ by the formula

$$
\begin{aligned}
F & =q \prod_{k=1}^{\infty}\left(1-q^{k}\right)\left(1-q^{23 k}\right) \\
& =\frac{1}{2}\left(\sum_{x, y \in \mathbf{Z}} q^{x^{2}+x y+6 y^{2}}-\sum_{x, y \in \mathbf{Z}} q^{2 x^{2}+x y+3 y^{2}}\right) \\
& =q-q^{2}-q^{3}+q^{6}+q^{8}-q^{13}-q^{16}+q^{23}-q^{24}+\cdots .
\end{aligned}
$$

The formula for $N_{p}(f)$ given above can be reformulated as:

$$
N_{p}(f)=a_{p}+1 \quad \text { for all primes } p .
$$

Note that the coefficients of $F$ are multiplicative: one has $a_{m m^{\prime}}=a_{m} a_{m^{\prime}}$ if $m$ and $m^{\prime}$ are relatively prime. The $q$-series $F$ is a newform of weight 1 and level 23 . The associated Dirichlet series is

$$
\sum_{m=1}^{\infty} \frac{a_{m}}{m^{s}}=\prod_{p}\left(1-\frac{a_{p}}{p^{s}}+\left(\frac{p}{23}\right) \frac{1}{p^{2 s}}\right)^{-1} .
$$

5.4. The case $n=4$. The discriminant of $f=x^{4}-x-1$ is -283 . The polynomial $f$ has two simple roots and one double root $\bmod 283$, hence $N_{283}(f)=3$. If $p \neq 283$, one has

$$
N_{p}(f)= \begin{cases}0 \text { or } 4 & \text { if } p \text { can be written as } x^{2}+x y+71 y^{2} \\ 1 & \text { if } p \text { can be written as } 7 x^{2}+5 x y+11 y^{2} \\ 0 \text { or } 2 & \text { if }\left(\frac{p}{283}\right)=-1\end{cases}
$$

(These cases correspond to the Frobenius substitution of $p$ being conjugate in $S_{4}$ to $(12)(34)$ or $1,(123),(1234)$ or (12) respectively.)

A complete determination of $N_{p}(f)$ can be obtained via a newform $F=$ $\sum_{m=0}^{\infty} a_{m} q^{m}$ of weight 1 and level 283 given in [5, p. 80, example 2]:

$F=q+\sqrt{-2} q^{2}-\sqrt{-2} q^{3}-q^{4}-\sqrt{-2} q^{5}+2 q^{6}-q^{7}-q^{9}+2 q^{10}+q^{11}+\sqrt{-2} q^{12}+\cdots$.

One has:

$$
N_{p}(f)=1+\left(a_{p}\right)^{2}-\left(\frac{p}{283}\right) \quad \text { for all primes } p \neq 283 .
$$

I do not know any closed formula for $F$, but one can give one for its reduction $\bmod 283$; see Notes. This is more than enough to determine the integers $N_{p}(f)$, since they are equal to $0,1,2$ or 4 . 
5.5. The case $n \geq 5$. Here the only known result seems to be that $f=x^{n}-x-1$ is irreducible (Selmer [15]) and that its Galois group is the symmetric group $S_{n}$. No explicit connection with modular forms (or modular representations) is known, although some must exist because of the Langlands program.

\section{NOTES}

1.1. Here is another interpretation of $c_{0}(f)$. Let $K=\mathbf{Q}[x] /(f)$ be the number field defined by $f$. We have $[K: \mathbf{Q}]=n \geq 2$. For every $d \geq 1$, let $a_{d}(K)$ be the number of the ideals $\mathfrak{a}$ of the ring of integers of $K$ with $N(\mathfrak{a})=d$. The zeta function of $K$ is the Dirichlet series

$$
\zeta_{K}(s)=\sum_{d \geq 1} \frac{a_{d}(K)}{d^{s}} .
$$

Using standard recipes in analytic number theory, one can show that Theorem 1 is equivalent to saying that $\zeta_{K}$ is lacunary: most of its coefficients are zero. More precisely, if we denote by $N_{K}(X)$ the number of $d \leq X$ with $a_{d}(K) \neq 0$, one has

$$
N_{K}(X) \sim c_{K} \frac{X}{(\log X)^{c_{0}(f)}} \quad \text { for } X \rightarrow \infty,
$$

where $c_{K}$ is a strictly positive constant (cf. Odoni [13] and Serre [16, §3.5]). As for Theorem 2, it can be reformulated as

$$
N_{K}(X)=O\left(\frac{X}{(\log X)^{1 / n}}\right) \quad \text { for } X \rightarrow \infty,
$$

with " $O$ " replaced by " $O$ " if $n$ is not a power of a prime.

1.2. Jordan's Theorem (Theorem 4). The standard proof of Theorem 4 relies on the fact that the stabilizer $H_{x}$ of a point $x$ of $X$ has $|G| / n$ elements, since $X \simeq G / H_{x}$. When $x$ runs through the $n$ points of $X$, the subgroups $H_{x}$ have at least one point in common, namely the element 1 . Hence, their union has at most $n \cdot\left|H_{x}\right|-(n-1)$ elements, i.e. at most $|G|-(n-1)$ elements. This shows that there are at least $n-1$ elements of $G$ which do not belong to any $H_{x}$, i.e. which have no fixed point. The interest of Theorem 5 is that it replaces the crude lower bound $n-1$ by $|G| / n$, which is close to being optimal.

Remark. Another way of stating Theorem 4 is:

Theorem $4^{\prime}$. If $H$ is a proper subgroup of a finite group $G$, there is a conjugacy class of $G$ which does not meet $H$.

In group-character language, this can be restated as:

Theorem $4^{\prime \prime}$. There exist two characters of $G$ which are distinct but have the same restriction to $H$.

In other words, the characters of $G$ cannot be detected by their restriction to a proper subgroup of $G$. One needs at least two such subgroups (such as, for $\mathbf{G L}_{2}\left(\mathbf{F}_{q}\right)$, a Borel subgroup and a non-split Cartan subgroup). This is quite different from the case of compact connected Lie groups, where just one maximal torus is enough. 
1.3. Theorem 5. Theorem 5 originated with a question of Lenstra, in relation with Theorem 2, See Boston et al. 2] for more on this story.

2. Burnside's Lemma. The first two proofs we offer are basically the same. Only their styles are different: analytic number theorists love to write $\sum 1$ and to permute summations, while combinatorists are fond of counting the elements of a set by mapping it into another one.

Note that Burnside's Lemma implies directly the weak form of Jordan's Theorem (Theorem 4 above). Indeed, since the mean value of $\chi(g)$ is 1 , and the element $g=1$ contributes $n>1$, there has to be some $g \in G$ with $\chi(g)<1$, hence $\chi(g)=0$.

Note also that Burnside's Lemma, combined with Chebotarev's Density Theorem, gives the following result:

If $f$ is as in $\S 1.1$, the mean value of $N_{p}(f)$ for $p \rightarrow \infty$ is equal to 1 . In other words:

$$
\sum_{p \leq X} N_{p}(f) \sim \pi(X) \quad \text { for } X \rightarrow \infty .
$$

This is due to Kronecker [10] and Frobenius [7], in the slightly weaker form where "natural density" is replaced by "analytic density".

3. Lifting circles to coverings. Theorem 3 does not extend to infinite coverings. Indeed, it is easy to construct an infinite free group $G$ having a subgroup $H$ of infinite index such that $\cup g H^{-1}=G$. If one chooses a connected graph $S$ with fundamental group isomorphic to $G$, the covering $T \rightarrow S$ associated with $H$ has the property that every continuous map $\mathbf{S}_{1} \rightarrow S$ can be lifted to $T$.

4. Chebotarev Density Theorem. The original proof can be found in 19]; it uses "analytic density" instead of "natural density". The more precise form we give was pointed out by Artin [1, even before Chebotarev's Theorem was proved.

For the history of this theorem, see [11, which also includes a sketch of a proof. For applications, see for instance [16] or [18.

Note that, for the application we make to Theorems 1 and 2 a weaker version of the theorem would be enough, namely the one proved by Frobenius [7] (with, once again, the proviso that "analytic density" has to be replaced by the "natural density").

5.1. Computation of $N_{p}(f)$. For a given polynomial $f$, such as $x^{3}-x-1$, $x^{4}-x-1$, etc., the numerical computation of $N_{p}(f)$ is an interesting question, especially for large values of the prime $p$. There are essentially two methods:

- The naive one is to try successively all the values of $x \bmod p$, and count those which are zeros of $f \bmod p$. This is slow; it requires exponential time (with respect to the number of digits of $p$ ); it is reasonable for very small primes only (up to 5 digits, say).

- The second method is much faster ("P" instead of "NP") and can handle primes of about 100 digits. It relies on the standard fact that computing $x^{p}$ by successive squarings takes about $\log p$ operations. One applies this principle to the finite $\mathbf{F}_{p}$-algebra $A_{p}=\mathbf{F}_{p}[X] /(f)$, 
with $x$ equal to the image of $X$ in $A_{p}$. Once $x^{p}$ is computed, one gets $N_{p}(f)$ by the formula:

$n-N_{p}(f)=$ rank of the linear endomorphism $u \mapsto\left(x^{p}-x\right) u$ of $A_{p}$.

Note that a variant of this method is incorporated in programs such as "PARI", where one has only to ask "polrootsmod $(f, p)$ ?" to get the list of the roots of $f \bmod p$.

5.2. $N_{p}(f)$ for $f=x^{2}-x-1$. For $p \neq 2,5$, the roots of $f_{p}$ in $\overline{\mathbf{F}}_{p}$ are $(1 \pm \sqrt{5}) / 2$; hence $N_{p}(f)=2$ if 5 is a square $(\bmod p)$, and $N_{p}(f)=0$ if not. By quadratic reciprocity, the first case occurs if and only if $p \equiv \pm 1(\bmod 5)$. A direct proof is as follows: call $z$ a primitive $5^{\text {th }}$ root of unity in $\overline{\mathbf{F}}_{p}$ and put $x=-\left(z+z^{4}\right), x^{\prime}=-\left(z^{2}+z^{3}\right)$. One has $x+x^{\prime}=1$ and $x x^{\prime}=-1$ because $1+z+z^{2}+z^{3}+z^{4}=0$. Hence, $x, x^{\prime}$ are the zeros of $f_{p}$. The action of the Frobenius $\sigma_{p}$ on $X=\left\{x, x^{\prime}\right\}$ is clear: we have $\sigma_{p}(x)=-\left(z^{p}+z^{-p}\right)$. If $p \equiv \pm 1(\bmod 5)$, we have $z^{p}=z^{ \pm 1}$, hence $\sigma_{p}(x)=x, \sigma_{p}\left(x^{\prime}\right)=x^{\prime}$, and $N_{p}(f)=2$; if $p \equiv \pm 2(\bmod 5)$, the same argument shows that $\sigma_{p}$ permutes $x$ and $x^{\prime}$, hence $N_{p}(f)=0$.

Remark. Even though the two cases $N_{p}(f)=0$ and $N_{p}(f)=2$ arise "equally often" (in an asymptotic sense, when $p \rightarrow \infty$ ), yet there is a definite bias towards the first case. This is an example of what Rubinstein and Sarnak call "Chebyshev Bias"; cf. [14].

5.3. $N_{p}(f)$ for $f=x^{3}-x-1$. Let $E=\mathbf{Q}[X] /(f)$ be the cubic field defined by $f$, and let $L$ be its Galois closure. We have $\operatorname{Gal}(L / \mathbf{Q})=S_{3}$. The field $L$ is a cubic cyclic extension of the quadratic field $K=\mathbf{Q}(\sqrt{-23})$; it is unramified, and, since $h(-23)=3$, it is the Hilbert class field of $K$, i.e. the maximal unramified abelian extension of $K$ (as a matter of fact, it is also the maximal unramified extension - abelian or not - of $K$, as follows from the Odlyzko bounds; see e.g. Martinet [12].)

If $p \neq 23$, let $\sigma_{p}$ be the Frobenius substitution of $p$ in $S_{3}=\operatorname{Gal}(L / \mathbf{Q})$; it is well-defined, up to conjugation. The image of $\sigma_{p}$ by sgn $: S_{3} \rightarrow\{ \pm 1\}$ is $\epsilon(p)$, where $\epsilon$ is the quadratic character associated with $K / \mathbf{Q}$, i.e., $\epsilon(p)=$ $\left(\frac{p}{23}\right)$. This shows that $\sigma_{p}$ is a transposition if $\left(\frac{p}{23}\right)=-1$, hence $N_{p}(f)=1$ in that case. When $\left(\frac{p}{23}\right)=1, \sigma_{p}$ is of order 1 or 3 , hence $N_{p}(f)=3$ or $N_{p}(f)=0$. To distinguish between these two cases, one decomposes $p$ in $K$ as $\mathfrak{p} \cdot \overline{\mathfrak{p}}$, and one has to decide whether $\mathfrak{p}$ is principal or not. The standard correspondence between ideal classes and binary quadratic forms shows that $\mathfrak{p}$ is principal is equivalent to $p$ being representable by the form $x^{2}+x y+6 y^{2}$, while $\mathfrak{p}$ is non-principal is equivalent to $p$ being representable by the form $2 x^{2}+x y+3 y^{2}$. This gives the recipe we wanted, namely,

$$
N_{p}(f)= \begin{cases}3 & \text { if } p \text { is representable by } x^{2}+x y+6 y^{2} \\ 0 & \text { if } p \text { is representable by } 2 x^{2}+x y+3 y^{2} \\ 1 & \text { if }\left(\frac{p}{23}\right)=-1\end{cases}
$$

The natural embedding $\rho$ of $S_{3}=\operatorname{Gal}(L / \mathbf{Q})$ in $\mathbf{G L}_{2}(\mathbf{C})$ gives rise to an Artin $L$-function

$$
L(\rho, s)=\sum_{m=1}^{\infty} \frac{a_{m}}{m^{s}}
$$


with coefficients $a_{m} \in \mathbf{Z}$. One may characterize it by

$$
L(\rho, s)=\zeta_{E}(s) / \zeta(s),
$$

where $\zeta_{E}(s)$ is the zeta function of the cubic field $E$. This is equivalent to saying that the linear representation $\rho \oplus 1$ is isomorphic to the 3 -dimensional permutation representation of $S_{3}$. By comparing the traces of $\sigma_{p}$ in both representations, we get $N_{p}(f)=a_{p}+1$ for every prime $p$ (including $p=23$ ). Since $S_{3}$ is a dihedral group, Hecke's theory applies and shows that the power series $F=\sum_{m=1}^{\infty} a_{m} q^{m}$ with the same coefficients as $L(\rho, s)$ is a cusp form of weight 1 and level 23, with respect to the character $\epsilon$. The explicit expressions of $F$ given in the text can be checked by standard modular methods.

5.4. $N_{p}(f)$ for $f=x^{4}-x-1$. Let $E$ be the quartic field defined by $f$ and $L$ its Galois closure; the Galois group $G=\operatorname{Gal}(L / \mathbf{Q})$ is isomorphic to $S_{4}$. Let $H$ be the unique normal $(2,2)$-subgroup of $G$; the quotient $G / H$ is isomorphic to $S_{3}$. The field $L^{H}$ is the Hilbert class field of $\mathbf{Q}(\sqrt{-283})$; note that $h(-283)=3$. The same argument as in Note 5.3 gives the image of the Frobenius $\sigma_{p}$ in $G / H$ in terms of $\left(\frac{p}{283}\right)$ and of the binary forms $x^{2}+x y+71 y^{2}$ and $7 x^{2}+5 x y+11 y^{2}$ with discriminant -283 .

To go further, one needs a result of Tate (reproduced in [17, 12], [5]) which says that the field $L$ has a quadratic extension $\widetilde{L}$ having the following two properties:

- $\widetilde{L}$ is unramified over $L$ (and hence also over $\mathbf{Q}(\sqrt{-283})$ );

- $\widetilde{L}$ is a Galois extension of $\mathbf{Q}$.

(An explicit construction of $\widetilde{L}$, due to Tate, is: $\widetilde{L}=L\left(\sqrt{4-7 x^{2}}\right)$, where $x$ is a root of $f$ in $L$; the construction given in Crespo 5 is more complicated.)

Martinet 12 has shown that $\widetilde{L}$ is the maximal unramified extension of $\mathbf{Q}(\sqrt{-283})$; in other words, the fundamental group of the ring $\mathbf{Z}[(1+\sqrt{-283}) / 2]$ is isomorphic to the "binary tetrahedral group" $\widetilde{A_{4}}=$ $\mathbf{S L}_{2}\left(\mathbf{F}_{3}\right)$.

The group $\widetilde{G}=\operatorname{Gal}(\widetilde{L} / \mathbf{Q})$ is isomorphic to $\mathbf{G L}_{2}\left(\mathbf{F}_{3}\right)$; it has a natural embedding $\rho$ in $\mathbf{G L}_{2}(\mathbf{C})$; its character has values in $\mathbf{Z}[\sqrt{-2}]$. By a wellknown theorem of Langlands and Tunnell (see the references in [5]), the $L$-series attached to $\rho$ corresponds to a modular form $F=\sum_{m=0}^{\infty} a_{m} q^{m}$ of weight 1 and level 283 whose first hundred coefficients are computed in [5]. One checks (by a character computation) that one has

$$
\rho \otimes \rho=\epsilon \oplus(\theta-1),
$$

where $\theta$ is the 4-dimensional permutation representation of $G$ and $\epsilon$ is the sign character of $G$. By taking traces, this gives

$$
\left(a_{p}\right)^{2}=\left(\frac{p}{283}\right)+N_{p}(f)-1, \quad \text { for all primes } p \neq 283 .
$$

Remark. One may give an explicit formula for $F(\bmod 283)$ as follows: by a known result [17, 9.3.1] $F$ is congruent $\bmod 283$ to a modular form $\varphi$ of weight $(283+1) / 2=142$, and of level 1 . Hence, $\varphi$ can be written as a linear combination, with coefficients in $\mathbf{F}_{283}$, of the standard basis:

$$
Q R^{23} \Delta, Q R^{21} \Delta^{2}, \ldots, Q R \Delta^{11}
$$


(with Ramanujan's notation:

$$
Q=1+240 \sum_{n=1}^{\infty} \frac{n^{3} q^{n}}{1-q^{n}}, \quad R=1-504 \sum_{n=1}^{\infty} \frac{n^{5} q^{n}}{1-q^{n}}
$$

and $\left.\Delta=\left(Q^{3}-R^{2}\right) / 1728=q \prod_{n=1}^{\infty}\left(1-q^{n}\right)^{24}\right)$.

A computation, using only the first eleven coefficients of $F$, gives the coefficients of $\varphi$ in that basis:

$$
[1,24,52,242,40,232,164,217,262,274,128] .
$$

In other words, we have

$$
F \equiv Q R^{23} \Delta+24 Q R^{21} \Delta^{2}+\cdots+128 Q R \Delta^{11}(\bmod 283) .
$$

(In these computations, I have selected 127 as $" \sqrt{-2} " \bmod 283$.)

\section{REFERENCES}

1. E. Artin, Über eine neue Art von L-Reihen, Hamb. Abh. 3 (1923), 89-108 (= Coll. Papers, 105-124)

2. N. Boston, W. Dabrowski, T. Foguel, P. J. Gies, D. A. Jackson, J. Leavitt and D. T. Ose, The proportion of fixed-point-free elements of a transitive permutation group, Comm. Algebra 21 (1993), 3259-3275. MR 94e:20002

3. W. Burnside, Theory of Groups of Finite Order, 2nd edition, Cambridge Univ. Press, 1911 (= Dover Publ., 1955). MR 16:1086c

4. P. J. Cameron and A. M. Cohen, On the number of fixed point free elements in a permutation group, Discrete Math. 106/107 (1992), 135-138. MR 93f:20004

5. T. Crespo, Galois representations, embedding problems and modular forms, Collectanea Math. 48 (1997), 63-83. MR 98j:11101

6. F. G. Frobenius, Über die Congruenz nach einem aus zwei endlichen Gruppen gebildeten Doppelmodul, J. Crelle 101 (1887), 279-299 (= Ges. Abh., II, 304-330).

7. F. G. Frobenius, Über Beziehungen zwischen den Primidealen eines algebraischen Körpers und den Substitutionen seiner Gruppe, Sitz. Akad. Wiss. Berlin (1896), 689-703 (= Ges. Abh., II, 719-733).

8. F. G. Frobenius, Über aufösbare Gruppen IV, Sitz. Akad. Wiss. Berlin (1901), 1216-1230 (= Ges. Abh., III, 189-203).

9. C. Jordan, Recherches sur les substitutions, J. Liouville 17 (1872), 351-367 (= Oe. I. 52).

10. L. Kronecker, Über die Irreductibilität von Gleichungen, Sitz. Akad. Wiss. Berlin (1880), 155-162 (= Werke, II, 83-93).

11. H. W. Lenstra, Jr., and P. Stevenhagen, Chebotarëv and his density theorem, Math. Intelligencer 18 (1996), 26-37. MR 97e:11144

12. J. Martinet, Petits discriminants des corps de nombres, Journées arithmétiques 1980 (J. V. Armitage, ed.), Cambridge Univ. Press, Cambridge, 1982, pp. 151-193. MR 84g:12009

13. R. W. K. Odoni, On the norms of algebraic integers, Mathematika 22 (1975), 71-80. MR 54:12715

14. M. Rubinstein and P. Sarnak, Chebyshev's Bias, Experiment. Math. 3 (1994), 173-197. MR 96d:11099

15. E. S. Selmer, On the irreducibility of certain trinomials, Math. Scand. 4 (1956), 287-302. MR 19:7f

16. J-P. Serre, Divisibilité de certaines fonctions arithmétiques, L'Ens. Math. 22 (1976), 227-260 (= Oe. 108). MR 55:7958

17. J-P. Serre, Modular forms of weight one and Galois representations, Algebraic Number Fields (A. Fröhlich, ed.), Acad. Press, London, 1977, pp. 193-268 (= Oe. 110). MR 56:8497 
18. J-P. Serre, Quelques applications du théorème de densité de Chebotarev, Publ. Math. I.H.E.S. 54 (1981), 123-201 (= Oe. 125). MR 83k:12011

19. N. Tschebotareff (Chebotarev), Die Bestimmung der Dichtigkeit einer Menge von Primzahlen, welche zu einer gegebenen Substitutionsklasse gehören, Math. Ann. 95 (1925), 191-228.

Collège de France, 3, Rue d'Ulm, Paris, France

E-mail address: serre@dmi.ens.fr 